\title{
Pseudodystonia: a new perspective on an old phenomenon
}

Rok Berlot, MD, PhD, ${ }^{\mathrm{a}}$ Kailash P. Bhatia, MD, FRCP, ${ }^{\mathrm{b}}$ Maja Kojović, MD, PhD ${ }^{\mathrm{a}^{*}}$

${ }^{a}$ Department of Neurology, University Medical Centre Ljubljana, Zaloška 2, 1000

Ljubljana, Slovenia

${ }^{\mathrm{b}}$ Sobell Department of Motor Neuroscience and Movement Disorders, UCL Institute of Neurology, Queen Square, London WC1N 3BG, UK

* Corresponding author:

Dr Maja Kojović

Department of Neurology, University Medical Centre Ljubljana

Zaloška 2, 1000 Ljubljana, Slovenia

Phone: +3861522 2311

Email: maja.kojovic@kclj.si 


\begin{abstract}
Pseudodystonia represents a wide range of conditions that mimic dystonia, including disorders of the peripheral nervous system, spinal cord, brainstem, thalamus, cortex and non-neurological conditions such as musculoskeletal diseases. Here, we propose a definition of pseudodystonia and suggest a classification based on underlying pathophysiological mechanisms. We describe phenomenology of different forms of pseudodystonia and point to distinctions between dystonia and pseudodystonia as well as challenging issues that may arise in clinical practice. The term pseudodystonia can be used to describe abnormal postures, repetitive movements or both, in which results of clinical, imaging, laboratory or electrophysiological investigations provide definite explanation of symptoms which is not compatible with dystonia. Pseudodystonia can be classified into non-neurological disorders of the musculoskeletal system, disorders of sensory pathways, disorders of motor pathways and compensatory postures in other neurological diseases. Presence of associated neurological findings in the affected body part is the key towards diagnosis of pseudodystonia. Additional supporting features are the presence of fixed postures, the absence of sensory trick, acute mode of onset and severe pain. Worsening on eye closure, traditionally considered typical for pseudodystonia, is not always present and can also appear in dystonia. It is challenging to separate dystonia and pseudodystonia in patients with thalamic lesions or corticobasal syndrome, where abnormal postures coexist with sensory loss. Many cases of pseudodystonia are treatable. Therefore, it is essential to consider pseudodystonia in a differential diagnosis of abnormal postures until a detailed neurological examination rules it out.
\end{abstract}




\section{Introduction}

Despite substantial advances in diagnostic techniques, patient observation remains a cornerstone in movement disorders. Dystonia is defined by sustained or intermittent muscle contractions causing abnormal, repetitive movements, postures or both [1]. Its diagnosis is commonly made at first sight. However, a common mimic of dystonia is a group of heterogeneous conditions referred to as pseudodystonia. Patients with pseudodystonia present with involuntary postures and patterned movements that comply with the definition of dystonia, but are due to other, identifiable causes. Thus, detailed neurological assessment and a broader differential diagnosis is required even in highly specialised movement disorder clinics [2].

Compared to other types of hyperkinetic movement disorders, such as tremor or chorea, dystonia has more "look-alikes". In addition, while common mimics of tremor or myoclonus can be differentiated on the basis of the definition, disorders presenting as pseudodystonia conform to the definition of dystonia. A main goal for the clinician is to organize many dystonias into a clinically meaningful syndromic pattern in order to facilitate diagnosis, treatment and clinical research. But, if not aware that many abnormal postures or repetitive movements might represent pseudodystonia, a clinician may contemplate the differential diagnosis of dystonia syndromes, while missing a treatable disorder imitating dystonia. In the updated classification of dystonia, the consensus acknowledged limitations of the current definitions of dystonia and pseudodystonia. One is arguably the definition of dystonia itself, as it does not require an exclusion of pseudodystonia. It was suggested that future definitions of dystonia might incorporate aspects of pathogenesis that exclude 
pseudodystonias. Albanese et al. provide a list of possible causes of pseudodystonia [1]. However, there is no detailed elaboration of pseudodystonia in the current literature.

In this review, we propose a definition of pseudodystonia and suggest classification of pseudodystonia based on underlying pathophysiological mechanisms, which can be applied for systematic approach in clinical practice. We describe phenomenology of different forms of pseudodystonia (with video examples) and discuss diverse etiologies. We point to "red flags" for distinction between dystonia and pseudodystonia and discuss challenging issues that may arise in clinical practice.

\section{Current definitions of pseudodystonia and ambiguities}

The prefix "pseudo-" in pseudodystonia comes from Greek language, meaning "lying, false". The term "pseudodystonia" suggests that a condition clinically resembles "true" dystonia, but is essentially something else. Fahn proposed to use the term "pseudodystonia" to describe "disorders that can mimic torsion dystonia, but are not generally considered to be a true dystonia" [3]. Within the revised classification of dystonic disorders, pseudodystonia is described as a condition that mimics dystonia, but has a known or presumed cause that is thought to differ from the causes of the broader dystonia group [1].

Dystonia is traditionally accepted to be a disorder of the basal ganglia, but recent studies have shaped a view that dystonia is a network disorder involving the cerebellum, thalamus, brainstem and cortex. In contrast, pseudodystonia has been associated with abnormalities in other regions, for example the spinal cord or 
peripheral nerves. The division between dystonia and pseudodystonia becomes less clear in cases with pathology at higher anatomical levels, such as the brainstem, thalamus or cortex. Hence, a definition of pseudodystonia should include presence of findings indicative of involvement of the functional system not considered to be implicated in dystonia.

\section{Proposed definition and classification}

We propose the following definition: Pseudodystonia is a term that describes abnormal postures, repetitive movements or both, in which results of clinical, imaging, laboratory or electrophysiological investigations provide definite explanation of symptoms which is not compatible with dystonia.

Our proposed definition builds on the current definition of dystonia [1]. However, in contrast to the established definition of dystonia, our suggested definition of pseudodystonia does not require sustained or intermittent muscle contractions to be a cause of abnormal postures. This allows inclusion of conditions that feature abnormal postures, e.g. atlanto-axial subluxation, but have no underlying muscle activity.

In the consensus paper, Albanese et al. stated that pseudodystonias "have a known or presumed cause that is thought to differ from the causes of the broader dystonia group" [1]. As causes of many types of dystonia are often unknown, it is difficult to provide a definition that would discriminate between dystonia and pseudodystonia based on etiology. However, the investigation of a patient with an abnormal posture can provide objective findings not compatible with dystonia. Hence, our proposed definition requires that clinical, laboratory, electrophysiological or imaging findings 
confirm the involvement of a system otherwise not considered causative of dystonia. This may be damage to the musculoskeletal system, impaired proprioception, peripheral nerve hyperexcitability or compensatory abnormal posture.

Pseudodystonia may manifest as abnormal sustained postures with superimposed movements or fixed postures. As almost any neuroanatomical structure may be implicated, a better insight into causes of abnormal postures may be gained by focusing on implicated functional systems. The distinction between dystonia and pseudodystonia is important, as they require a different diagnostic and treatment approach. Similarly, it is important to recognise different causes of pseudodystonia. For example, cases of pseudodystonia due to osteomuscular involvement do not need further neurological workup, while many cases of pseudodystonia due to proprioceptive loss are reversible with specific treatment. In this respect, a classification of pseudodystonia based on pathophysiology may be warranted.

We propose classification of pseudodystonia in four categories: (i) non-neurological disorders of the musculoskeletal system; (ii) disorders of sensory pathways; (iii) disorders of motor pathways; and (iv) compensatory postures (Table 1). Such classification might not be absolute, but provides a possible starting point in the differential diagnosis among conditions that are often not considered together.

While the term dystonia is used by clinicians in different contexts, i.e. to refer to phenomenology of an involuntary movement, a syndrome with heterogenous etiology, or the idiopathic form of dystonia, we propose that the term pseudodystonia is used to describe phenomenology (what can be seen on examination), followed by 
pathophysiological explanation for the sign, e.g. pseudodystonic hands posturing caused by proprioceptive loss.

\subsection{Non-neurological disorders of the musculoskeletal system}

Musculoskeletal conditions can lead to sustained deformities manifesting as abnormal postures. Mutation in BIN1, a rare cause of centronuclear myopathy, was recently reported to manifest with severe rigidity of the spine alongside proximal limb and axial weakness [4]. Acquired or congenital atlanto-axial displacements such as Klippel-Feil syndrome may mimic cervical dystonia [3,5]. Another example of cervical pseudodystonia is congenital muscular torticollis, a rare congenital musculoskeletal disorder caused by unilateral shortening of the sternocleidomastoid muscles, which leads to ipsilateral head tilt and contralateral rotation of the face and chin [6]. Soft tissue mass in the cervical area, such as a retropharyngeal abscess, may irritate neck muscles resulting in postures mimicking cervical dystonia [7]. Torticollis may also be associated with gastroesophageal reflux in children with Sandifer syndrome, where positioning of the head possibly provides relief from abdominal discomfort caused by acid reflux [7]. Abnormal postures of hands may be seen in rheumatic disorders of the small joints and tendon alterations. In trigger finger, inflammation and hypertrophy of the tendon sheath progressively restrict the motion of the flexor tendon. This leads to clicking associated with movement of the finger, progressive loss of full flexion or extension and, ultimately, to a locked position in flexion [8]. Thickening of the palmar fascia in patients with Dupuytren's contracture leads to characteristic hand deformities in which joints of one or more fingers cannot be fully extended, resulting in a progressive flexion deformity (Fig. 1) [9]. In lower limbs, hip dislocation can cause pseudo-dystonic leg postures [10]. 


\subsection{Disorders of the sensory pathways}

The causative relationship between proprioceptive loss and pseudodystonia is supported by observations that the movement disorder is confined to the region of sensory loss and coincides with the onset and the resolution of sensory impairment [11]. Pseudodystonia can occur with lesions anywhere along the sensory pathways, from peripheral nerves to sensory cortex. It has been described in patients with parietal or thalamic stroke [11-13], medullar and pontine haemorrhage [14], syringomyelia [15], posttraumatic myelopathy [16], posterior column pathology in cases of cervical myelitis and cervical spondylotic myelopathy $[17,18]$, dorsal root ganglion neuronopathies [11], sensory ataxic polyneuropathy [19], cubital tunnel syndrome [11] and damage to lingual afferent fibres [20].

Pseudodystonia associated with deafferentation is important to recognize, because many of the underlying disorders are treatable. Perhaps the most illustrative example is the 'useless hand of Oppenheim', characterized by abnormal postures of the hand due to proprioceptive sensory loss (Video 1). It is typically caused by a demyelinating plaque in posterior columns of the cervical spinal cord and may be the first manifestation of multiple sclerosis $[21,22]$. The term should not be confused with the useless hand in the corticobasal syndrome, where various combinations of apraxia, dystonia, rigidity and motor neglect contribute to loss of useful hand function [23].

Pseudodystonia is also common in subacute combined degeneration (Video 2), where it can result from a combination of peripheral nerve and posterior column damage and improves with B12 supplementation [24,25]. Pseudodystonic postures due to 
proprioceptive loss may be characteristic of Miller-Fisher syndrome (Fig. 2, Video 3) or chronic inflammatory demyelinating polyradiculoneuropathy (Video 4) [26] and may respond to immunoglobulins or corticosteroids. In cases of polyneuropathy, the anatomical distribution of the posturing or abnormal movements provides an important clue to the underlying etiology, as pseudodystonia is almost exclusively distal, affecting hands and feet. Pseudodystonia in peripheral neuropathy may coexist with pseudoathetosis and neuropathic tremor in the same distribution.

Cases of pseudodystonia due to loss of proprioception hint at potential similar underlying mechanisms with dystonia, where subclinical sensory deficits have been demonstrated in a number of research studies. Possible mechanisms of involvement of sensory system in pathogenesis of dystonia might include defective processing of sensory information and failure to integrate sensory and motor inputs [27]. However, obvious sensory deficits cannot typically be demonstrated in individual patients with dystonia on clinical examination or with commonly used investigations. According to our proposed definition, cases where sensory deficits can be demonstrated objectively and are of sufficient magnitude to provide definite explanation of symptoms point towards pseudodystonia rather than dystonia.

\subsection{Disorders of the motor pathways}

While dystonia may be viewed as an abnormality of the network involved in movement preparation, resulting in formation of abnormal motor commands, pseudodystonia may arise when appropriate motor commands have been formed, but lower level structures that conduct neural impulses for the execution of movement are 
affected by disease. This may include disorders of the corticospinal and other descending motor tracts, the spinal cord, motor nerves or muscles.

Involuntary postures frequently accompany pyramidal tract involvement in patients with hemispheric or capsular stroke. Historically, Denny-Brown coined the term 'spastic dystonia' to describe tonic involuntary muscle contraction in the context of corticospinal tract injury [28]. Five types of abnormal arm posture may be seen following stroke, the most common being internal rotation/adduction of the shoulder, flexion of the elbow and neutral position of the arm and the wrist [29]. In addition to the involuntary tonic posturing, the same patients manifest other clear signs of pyramidal tract involvement (i.e. spasticity, increased deep tendon reflexes, Babinski sign and weakness). In this context, the term spastic dystonia is misnomer and abnormal posture caused by pyramidal tract involvement should be regarded as pseudodystonia. The term spastic dystonia is also commonly used among clinicians when spasticity and dystonia coexist in the same anatomical region, for example in cerebral palsy or in neurodegenerative diseases. Strictly speaking, the term spastic dystonia is misnomer also in this context, but probably came in the common use because it may not be easy to clinically distinguish between the components of spasticity and dystonia in the same patient. For example, spasticity may be more pronounced on sitting than in supine position and even more on walking, which resembles action-induced worsening of dystonia [30,31]. In general, the two key distinguishing features are velocity-dependent increase of tone with spastic catch, typical for spasticity and spastic dystonia, and worsening of abnormal postures with voluntary movement of a distant body part, which is characteristic of dystonia [32]. 
Stiff-person syndrome is characterized by sustained muscular contractions caused by abnormal excitability of spinal interneuronal networks [33]. Patients have characteristic gait with marked hyperlordosis, caused by axial rigidity [34]. However, symptoms may be confined to one limb only, as in stiff limb syndrome, mimicking limb dystonia (Fig. 3, Video 5), or may even present in hemidistribution, resembling hemidystonia [35].

Hyperexcitation of the pyramidal tract or peripheral motor nerves may be confused with paroxysmal dystonia. Painful tonic spasms (PTS) feature sudden-onset unilateral (rarely bilateral) stereotyped limb posturing, lasting few seconds, rarely up to 2 minutes and repeating several times per hour. PTS are most commonly associated with multiple sclerosis and neuromyelitis optica and may be the first manifestation of the disease [36]. Although PTS are frequently referred to in the literature as dystonic spasms or dystonic limb posturing, the pathophysiological mechanism is different from dystonia. PTS are caused by demyelinating lesions of the corticospinal pathway at the level of the posterior limb of the internal capsule or within the cerebral peduncle, where closely packed motor fibres allow radial spread of ephaptic activation [36,37]. Carpopedal spasms in hypocalcemia may resemble dystonic arm or foot posturing and are caused by repetitive axonal discharges due to increased neural excitability (Video 6). Neuromyotonia presents with muscle cramps and impaired muscle relaxation that may resemble dystonia [38]. However, the clue toward diagnosis of neuromyotonia is observation of myokymia - continuous, undulating, wave-like rippling of muscles that looks like a bag of worms under the skin. Needle EMG will reveal continuous motor unit activity, occurring as doublet, triplet or multiplet single motor unit discharges, firing at high intraburst frequency. Myotonia 
can lead to abnormal limb postures triggered by action or percussion, which is due to impaired and prolonged relaxation of the muscle. It is seen in myotonic dystrophy or congenital myotonia. Involuntary movements resembling dystonia may sometimes dominate the clinical picture of multifocal motor neuropathy, presumably due to spontaneous axonal impulse generation [39].

Any kind of muscle weakness (with or without superimposed contractures) may result in abnormal posturing, i.e. hand of benediction in median nerve palsy, claw hand in ulnar nerve palsy or ape hand in ALS.

\subsection{Compensatory postures in other neurological diseases}

Pseudodystonia may be a compensatory phenomenon, appearing as part of a distinct neurological disorder. For example, head tilt or head rotation may prevent diplopia in patients with trochlear or abducens nerve palsy, respectively [40] (Fig. 4). Head tilt is also a part of the ocular tilt reaction in the setting of injury to the static (otolithic) vestibular system or its central pathways. The ocular tilt reaction consists of head tilt, ipsiversive eye rotation and vertical eye divergence. It serves to restore subjective vertical orientation [41]. The common causes of head tilt are brainstem stroke in adults and posterior fossa tumours in children. In these cases, diplopia or vertigo are presenting symptoms, but clear history might be missing in some pediatric patients or adults with limited ability to communicate. A possible explanation for head tilt in posterior fossa space occupying lesions that do not cause double vision is compensatory effort to relieve the pressure by enlarging the surface area of the foramen magnum [42]. However, such lesions might also interfere with projections 
to/from the basal ganglia and the cerebellum, which would be compatible with "true" dystonia.

\section{How to distinguish between dystonia and pseudodystonia?}

Pseudodystonia can present either as focal, segmental or generalized (Table 2), mimicking dystonia in different distributions. We suggest several "red flags" for distinguishing between dystonia and pseudodystonia:

\subsection{Associated findings in the affected part of the body.}

Pseudodystonia usually features additional neurological findings in the same distribution. There could be evidence of motor nerve palsy (e.g. in torticollis due to abducens/trochlear nerve palsy) or nerve hyperexcitability (e.g. Trousseau sign in hypocalcemia - hand and arm spasms provoked by transient ischaemia). Sensory loss may be evident in peripheral neuropathies or spinal cord lesions.

\subsection{Fixed vs. mobile postures}

Pseudodystonia may be characterised by reduced active or passive range of movements (e.g. in osteomuscular torticollis), resulting in fixed postures, equally present at rest or action. In contrast, patients with dystonia experience mobile, actioninduced abnormal postures with dystonia overflow to "unaffected" muscles initiated by movement. It should be kept in mind that some secondary dystonias due to focal basal ganglia lesions or neurodegeneration may lead to contractures and fixed postures [43], while pseudodystonia due to proprioceptive loss features movements and mobile postures. 


\subsection{Worsening on eye closure}

It is considered that eye closure typically accentuates pseudodystonia caused by proprioceptive loss $[15,17]$. This has been attributed to compensation of abnormal posture by the visual system. When visual inputs are not available to guide corrective movements, pseudodystonia may get worse. However, eye closure had no apparent effect on the severity of movements in many reported cases of pseudodystonia $[11,16,44]$. Similarly, reopening of the eyes does not necessarily lead to correction of abnormal posture (Video 4). Moreover, while it has not been emphasised in the literature so far, "true" dystonia may also become more apparent with eye closure, presumably due to distraction (Video 7). Worsening of involuntary movements when attention is driven away may reflect failure of "top-down" attentional monitoring, by which higher-level cognitive centres control inappropriate movement caused by lower-level neural processing [45]. Hence, the effect of eye closure is not a reliable indicator of pseudodystonia. In cases where eye closure leads to worsening of abnormal movements, pseudodystonia must be confirmed by demonstrating proprioceptive deficits in relevant anatomical distribution on clinical examination.

\subsection{Absence of a sensory trick}

The sensory trick (alleviating maneuver) is a typical feature of isolated dystonia and its absence may suggest pseudodystonia. Again, it should be noted that the sensory trick is usually absent in secondary forms of dystonia [46].

\subsection{The mode of onset}


Dystonia rarely presents acutely while pseudodystonia often does. Notable exceptions among dystonias are acute dystonic reactions provoked by dopaminergic blocking agents and rapid onset dystonia-parkinsonism.

\subsection{Pain}

Severe pain is not a typical feature of dystonia. It is more commonly associated with pseudodystonia, particularly due to musculoskeletal involvement, stiff-person syndrome or painful tonic spasms of multiple sclerosis and neuromyelitis optica. Nevertheless, pain does accompany some forms of focal dystonia. It is a characteristic feature in patients with cervical dystonia [47]. Also, corneal pain may precede the onset of blepharospasm [48]. Pain also may accompany temporomandibular joint dysfunction caused by oromandibular dystonia [49].

\section{Challenging issues}

Thalamic lesions are commonly invoked in acquired dystonia [50]. However, thalamic damage may also cause reduced sensation of the contralateral side of the body, including loss of proprioception $[13,15]$. Possibly, many cases of thalamic and parietal lesions associated with sensory loss have been misclassified as dystonia instead of pseudodystonia. A similar issue arises regarding dystonia in corticobasal syndrome, where true dystonia may reflect basal ganglia involvement. However, patients with alien limb syndrome due to parietal lobe involvement probably have pseudodystonia. In alien limb syndrome, abnormal non-purposeful movement, i.e. arm levitation, may be a consequence of impaired proprioceptive feedback necessary for movement control, while feeling of the limb being "alien" is due to coexisting neglect of the same limb [51]. In our opinion, cases of abnormal posture with 
associated sensory deficits are better classified as pseudodystonia. This distinction may be important for studying pathophysiology of dystonia, where it is not desirable to lump together patients with different underlying mechanisms.

The term pseudoathetosis has been used to characterize slow sinuous, writhing movements of hands and fingers due to sensory loss that resemble athetosis $[52,53]$. However, athetosis is not a distinct type of movement disorder [54], but rather represents a form of very mobile dystonia, due to higher degree of freedom and reduced stiffness of hands and feet compared to more proximal joints of the limbs and the trunk [55]. The term pseudoathetosis is firmly established in the literature, in particular due to its specific phenomenology. However, it should be noted that sensory loss underlies both pseudoathetosis, manifesting as slow sinusoid writhing movements, and pseudodystonia, which presents with abnormal posturing with or without superimposed movements.

Abnormal limb postures in Parkinson's disease and atypical parkinsonism known as striatal hand and striatal foot represent fixed deformities that are typically present at rest (irrespective of activation of the limb), persist in sleep and are clearly associated with rigidity [56]. While these characteristics are different from features of isolated dystonia, it may be matter of debate whether to consider striatal limb deformities as secondary dystonia or pseudodystonia.

Arthrogryposis, characterised by multiple congenital contractures from various etiologies, has been traditionally viewed as pseudodystonia. However, recent studies have identified cases of biallelic DYT1 mutations presenting with arthrogryposis 
upon birth. Therefore, at least some cases of artrogryphosis share the etiology with DYT1 early-onset isolated dystonia and may represent a final stage of severe dystonia with contractures [57-59]. Indeed, further insight into the pathophysiology of dystonia and different forms of pseudodystonia will probably lead to reclassification of some conditions between the two groups.

Another challenging issue is whether to classify psychogenic (functional) dystonia as acquired dystonia or pseudodystonia. Functional dystonia often shares some features with pseudodystonia, such as acute onset and fixed postures. In the consensus update on phenomenology and classification of dystonia, Albanese et al. acknowledged there was an issue on how to categorize functional dystonia. Ultimately, the panel reached a consensus to classify it among acquired dystonias [1], although pathophysiology remains obscure and there is lack of evidence for basal ganglia or cerebellar involvement.

Another difficult issue concerns peripheral trauma induced dystonia, which is still an ambiguous entity [60]. In peripheral dystonias, the severity of trauma and the interval between trauma and emergence of abnormal postures varies considerably, alongside reported clinical characteristics of abnormal postures. It follows that peripheral dystonia is a heterogeneous group of conditions, likely encompassing several different entities. It is probable that in many cases the co-occurrence of peripheral trauma and dystonia was by chance. These patients have characteristics of dystonia that do not differ from characteristics of isolated dystonia, with the previous trauma being most probably coincidental $[61,62]$. On the other hand, many reported cases have acute to subacute onset of painful and fixed dystonic postures with absence of alleviating 
maneuvers, some featuring complex regional pain syndrome, suggesting these patients are manifesting functional (psychogenic) dystonia [63-65]. As discussed above, it might be a matter of debate whether functional dystonia is a "true" dystonia or pseudodystonia. However, considering that functional dystonias are currently classified among acquired dystonias, the subgroup of patients with fixed painful postures after trauma belong to the functional dystonia group. However, some patients with peripheral injury induced abnormal postures and clinical and electrophysiological findings of peripheral nerve damage may fit into the pseudodystonia group [66]. Finally, it remains unclear if in a proportion of patients another mechanism may play a role, such as peripheral trauma triggering reorganisation in sensory-motor circuits leading to functional changes in the brain network as postulated in isolated dystonia [60].

\section{Conclusions}

Pseudodystonia can be associated with a wide array of neurological and nonneurological disorders. It is most commonly described in patients with sensory deficits, where defective processing of proprioceptive information might lead to inability to integrate sensory and motor inputs in the striatum or in the sensorimotor cortex. However, many conditions with varied pathophysiology can lead to pseudodystonic postures. Therefore, it is essential to keep pseudodystonia in mind as a differential diagnosis, because many cases are treatable. The clinical approach to pseudodystonia is likely to be aided with our proposed definition and classification of pseudodystonias, which however require scrutiny from the clinical and research communities before they can be generally accepted. Early recognition and correct 
identification of the underlying etiology of pseudodystonia can lead to complete recovery.

\section{Ethics and conflict of interest}

All patients provided informed, written consent to publish the photographs and videos in print and online. The authors declare no conflict of interests. This study did not receive any specific grant from funding agencies in the public, commercial, or nonfor-profit sectors.

\section{References}

[1] A. Albanese, K. Bhatia, S.B. Bressman, M.R. Delong, S. Fahn, V.S.C. Fung, M. Hallett, J. Jankovic, H.A. Jinnah, C. Klein, A.E. Lang, J.W. Mink, J.K. Teller, Phenomenology and classification of dystonia: A consensus update, Mov. Disord. 28 (2013) 863-873.

[2] B.R. Bloem, N.C. Voermans, M.B. Aerts, K.P. Bhatia, B.G.M. van Engelen, B.P. van de Warrenburg, The wrong end of the telescope: neuromuscular mimics of movement disorders (and vice versa), Pract. Neurol. 16 (2016) 2649.

[3] S. Fahn, S.B. Bressman, C.D. Marsden, Classification of dystonia., Adv. Neurol. 78 (1998) 1-10.

[4] M. Cabrera-Serrano, F. Mavillard, V. Biancalana, E. Rivas, B. Morar, A. Hernández-Laín, M. Olive, N. Muelas, E. Khan, A. Carvajal, P. Quiroga, J. Diaz-Manera, M. Davis, R. Ávila, C. Domínguez, N.B. Romero, J.J. Vílchez, D. Comas, N.G. Laing, J. Laporte, L. Kalaydjieva, C. Paradas, A Roma founder BIN1 mutation causes a novel phenotype of centronuclear myopathy 
with rigid spine., Neurology. 91 (2018) e339-e348.

doi:10.1212/WNL.0000000000005862.

[5] M. Lopez-Vicchi, G. Da Prat, E.M. Gatto, Pseudodystonic Posture Secondary to Klippel-Feil Syndrome and Diastematomyelia., Tremor Other Hyperkinet. Mov. (N. Y). 5 (2015) 325. doi:10.7916/D8ZC820C.

[6] K. Nilesh, S. Mukherji, Congenital muscular torticollis., Ann. Maxillofac. Surg. 3 (2013) 198-200.

[7] A. Tumturk, G. Kaya Ozcora, A. Kacar Bayram, M. Kabaklioglu, S. Doganay, M. Canpolat, H. Gumus, S. Kumandas, E. Unal, A. Kurtsoy, H. Per, Torticollis in children: an alert symptom not to be turned away, Child's Nerv. Syst. 31 (2015) 1461-1470.

[8] A.H. Makkouk, M.E. Oetgen, C.R. Swigart, S.D. Dodds, Trigger finger: etiology, evaluation, and treatment., Curr. Rev. Musculoskelet. Med. 1 (2008) 92-6.

[9] W.A. Townley, R. Baker, N. Sheppard, A.O. Grobbelaar, Dupuytren's contracture unfolded., BMJ. 332 (2006) 397-400.

[10] S.R. Chandra, T.G. Issac, Battered woman syndrome: An unusual presentation of pseudodystonia., J. Neurosci. Rural Pract. 5 (2014) 189-90.

[11] F.R. Sharp, T.A. Rando, S.A. Greenberg, L. Brown, S.M. Sagar, Pseudochoreoathetosis. Movements associated with loss of proprioception., Arch. Neurol. 51 (1994) 1103-9.

[12] F. Salih, C. Zimmer, H. Meierkord, Parietal proprioceptive loss with pseudoathetosis [5], J. Neurol. 254 (2007) 396-397.

[13] J.S. Kim, Delayed onset mixed involuntary movements after thalamic stroke: clinical, radiological and pathophysiological findings., Brain. 124 (2001) 299- 
309.

[14] L. Torres, C. Cosentino, R. Suárez, Pseudoathetosis after medullar and pontine hemorrhage, Rev. Neurol. 34 (2002) 89-90.

[15] M. Spitz, A.A. Costa Machado, R. do Carmo Carvalho, F. Martins Maia, M.S. Haddad, D. Calegaro, M. Scaff, E.R. Barbosa, Pseudoathetosis: Report of three patients, Mov. Disord. 21 (2006) 1520-1522.

[16] K. Bray, S.K. Chhetri, A. Varma, M. Silverdale, Reversible pseudoathetosis induced by cervical myelopathy, Mov. Disord. 27 (2012) 1370-1371.

[17] J. Pujol, J. Monells, E. Tolosa, J.M. Soler-Insa, J. Valls-Solé, Pseudoathetosis in a patient with cervical myelitis: neurophysiologic and functional MRI studies., Mov. Disord. 15 (2000) 1288-93.

[18] W.-J. Hwang, Reversible pseudoathetosis and sensory ataxic gait caused by cervical spondylotic myelopathy, J. Clin. Neurosci. 34 (2016) 271-272.

[19] Y.L. Lo, S. See, Pseudoathetosis, N. Engl. J. Med. 363 (2010) e29. doi:10.1056/NEJMicm0907786.

[20] R.W. Orrell, C.D. Marsden, The neck-tongue syndrome., J. Neurol. Neurosurg. Psychiatry. 57 (1994) 348-352.

[21] R.J. Coleman, L. Russon, K. Blanshard, S. Currie, Useless hand of Oppenheim--magnetic resonance imaging findings., Postgrad. Med. J. 69 (1993) 149-50.

[22] L. Wiblin, J. Guadagno, The useless hand of Oppenheim, Pract. Neurol. 17 (2017) 464-468.

[23] M. Kojović, K.P. Bhatia, Bringing order to higher order motor disorders, J. Neurol. (2018). doi:10.1007/s00415-018-8974-9.

[24] S. Blunt, M. Silva, C. Kennard, R. Wise, Vitamin B12 deficiency presenting 
with severe pseudoathetosis of upper limbs., Lancet. 343 (1994) 550.

[25] G. Sciacca, F. Le Pira, G. Mostile, A. Nicoletti, M. Zappia, Toe pseudoathetosis in vitamin B12 deficiency., Eur. J. Neurol. 23 (2016) e30-1. doi:10.1111/ene.12972.

[26] K. Ng, MADSAM neuropathy: An unusual cause of pseudoathetosis, Neurology. 83 (2014) 291.

[27] M. Hallett, Is dystonia a sensory disorder?, Ann. Neurol. 38 (1995) 139-40.

[28] D. Denny-Brown, The nature of dystonia, Bull. N. Y. Acad. Med. 41 (1965) 858-69.

[29] H. Hefter, W.H. Jost, A. Reissig, B. Zakine, A.M. Bakheit, J. Wissel, Classification of posture in poststroke upper limb spasticity: a potential decision tool for botulinum toxin A treatment?, Int. J. Rehabil. Res. 35 (2012) $227-33$.

[30] K. Irmady, B. Jabbari, E.D. Louis, Arm Posturing in a Patient Following Stroke: Dystonia, Levitation, Synkinesis, or Spasticity?, Tremor Other Hyperkinet. Mov. (N. Y). 5 (2015) 353. doi:10.7916/D8222TBH.

[31] A. Thibaut, C. Chatelle, E. Ziegler, M.-A. Bruno, S. Laureys, O. Gosseries, Spasticity after stroke: physiology, assessment and treatment., Brain Inj. 27 (2013) 1093-105.

[32] A. Jethwa, J. Mink, C. Macarthur, S. Knights, T. Fehlings, D. Fehlings, Development of the Hypertonia Assessment Tool (HAT): a discriminative tool for hypertonia in children., Dev. Med. Child Neurol. 52 (2010) e83-7.

[33] A. McKeon, M.T. Robinson, K.M. McEvoy, J.Y. Matsumoto, V.A. Lennon, J.E. Ahlskog, S.J. Pittock, Stiff-man syndrome and variants: clinical course, treatments, and outcomes., Arch. Neurol. 69 (2012) 230-8. 
[34] J.F. Baizabal-Carvallo, J. Jankovic, Autoimmune and paraneoplastic movement disorders: An update, J. Neurol. Sci. 385 (2018) 175-184.

[35] J. Kenda, M. Kojović, F. Graus, M. Gregorič Kramberger, (Pseudo)hemidystonia associated with anti-glutamic acid decarboxylase antibodies--a case report., Eur. J. Neurol. 22 (2015) 1573-4.

[36] G. Deuschl, Movement disorders in multiple sclerosis and their treatment., Neurodegener. Dis. Manag. 6 (2016) 31-35.

[37] A. Spissu, A. Cannas, P. Ferrigno, A.E. Pelaghi, M. Spissu, Anatomic correlates of painful tonic spasms in multiple sclerosis., Mov. Disord. 14 (1999) 331-5.

[38] H.L. Geyer, S.B. Bressman, The diagnosis of dystonia, Lancet Neurol. 5 (2006) 780-790.

[39] N. Garg, R.N.S. Heard, L. Kiers, R. Gerraty, C. Yiannikas, Multifocal Motor Neuropathy Presenting as Pseudodystonia, Mov. Disord. Clin. Pract. 4 (2017) 100-104. doi:10.1002/mdc3.12336.

[40] J. Varrato, S. Galetta, Fourth nerve palsy unmasked by botulinum toxin therapy for cervical torticollis., Neurology. 55 (2000) 896.

[41] K.H. Cho, Y.D. Kim, J. Kim, B.S. Ye, J.H. Heo, H.S. Nam, Contraversive ocular tilt reaction after the lateral medullary infarction., Neurologist. 19 (2015) 79-81.

[42] J.E. Piña-Garza, Increased intracranial pressure, in: Fenichel's Clinical Pediatric Neurology: A signs and symptoms approach. 7th ed. Saunders, London, 2013, pp. 89-112.

[43] A. Schrag, M. Trimble, N. Quinn, K. Bhatia, The syndrome of fixed dystonia: an evaluation of 103 patients., Brain. 127 (2004) 2360-72. 
[44] J. Ghika, J. Bogousslavsky, Spinal pseudoathetosis: a rare, forgotten syndrome, with a review of old and recent descriptions., Neurology. 49 (1997) 432-7.

[45] N.S. Narayanan, M. Laubach, Top-down control of motor cortex ensembles by dorsomedial prefrontal cortex., Neuron. 52 (2006) 921-31.

[46] V.F.M.L. Ramos, B.I. Karp, M. Hallett, Tricks in dystonia: ordering the complexity., J. Neurol. Neurosurg. Psychiatry. 85 (2014) 987-93.

[47] P.D. Charles, C.H. Adler, M. Stacy, C. Comella, J. Jankovic, A. Manack Adams, M. Schwartz, M.F. Brin, Cervical dystonia and pain: characteristics and treatment patterns from CD PROBE (Cervical Dystonia Patient Registry for Observation of OnabotulinumtoxinA Efficacy)., J. Neurol. 261 (2014) 1309-19.

[48] D. Borsook, P. Rosenthal, Chronic (neuropathic) corneal pain and blepharospasm: Five case reports, Pain. 152 (2011) 2427-2431.

[49] L. Slaim, M. Cohen, P. Klap, M. Vidailhet, A. Perrin, D. Brasnu, D. Ayache, M. Mailly, Oromandibular Dystonia: Demographics and Clinical Data from 240 Patients., J. Mov. Disord. 11 (2018) 78-81.

[50] M.S. Lee, C.D. Marsden, Movement disorders following lesions of the thalamus or subthalamic region., Mov. Disord. 9 (1994) 493-507.

[51] J. Graff-Radford, M.N. Rubin, D.T. Jones, A.J. Aksamit, J.E. Ahlskog, D.S. Knopman, R.C. Petersen, B.F. Boeve, K.A. Josephs, The alien limb phenomenon, J. Neurol. 260 (2013) 1880-1888.

[52] J.G.L. Morris, S.K. Jankelowitz, V.S.C. Fung, P.D. Clouston, M.W. Hayes, P. Grattan-Smith, Athetosis I: historical considerations., Mov. Disord. 17 (2002) $1278-80$.

[53] J.G.L. Morris, P. Grattan-Smith, S.K. Jankelowitz, V.S.C. Fung, P.D. 
Clouston, M.W. Hayes, Athetosis II: the syndrome of mild athetoid cerebral palsy., Mov. Disord. 17 (2002) 1281-7.

[54] D.J. Lanska, Early Controversies over Athetosis: I. Clinical Features, Differentiation from other Movement Disorders, Associated Conditions, and Pathology., Tremor Other Hyperkinet. Mov. (N. Y). 3 (2013). doi:10.7916/D8TT4PPH.

[55] J.A. Obeso, Commentary for reversible pseudoathetosis induced by cervical myelopathy., Mov. Disord. 27 (2012) 1371.

[56] R. Ashour, R. Tintner, J. Jankovic, Striatal deformities of the hand and foot in Parkinson's disease., Lancet. Neurol. 4 (2005) 423-31.

[57] A. Kariminejad, M. Dahl-Halvarsson, G. Ravenscroft, F. Afroozan, E. Keshavarz, H. Goullée, M.R. Davis, M. Faraji Zonooz, H. Najmabadi, N.G. Laing, H. Tajsharghi, TOR1A variants cause a severe arthrogryposis with developmental delay, strabismus and tremor, Brain. 140 (2017) 2851-2859.

[58] S.C. Reichert, P. Gonzalez-Alegre, G.H. Scharer, Biallelic TORIA variants in an infant with severe arthrogryposis, Neurol. Genet. 3 (2017) e154. doi:10.1212/NXG.0000000000000154.

[59] E. Isik, A. Aykut, T. Atik, O. Cogulu, F. Ozkinay, Biallelic TOR1A mutations cause severe arthrogryposis: A case requiring reverse phenotyping, Eur. J. Med. Genet. (2018). doi:10.1016/j.ejmg.2018.09.011.

[60] H. Kumar, M. Jog, Peripheral trauma induced dystonia or post-traumatic syndrome?, Can. J. Neurol. Sci. 38 (2011) 22-9.

[61] A. Samii, P.K. Pal, M. Schulzer, E. Mak, J.K. Tsui, Post-traumatic cervical dystonia: a distinct entity?, Can. J. Neurol. Sci. 27 (2000) 55-9.

[62] C. Sankhla, E.C. Lai, J. Jankovic, Peripherally induced oromandibular 
dystonia., J. Neurol. Neurosurg. Psychiatry. 65 (1998) 722-8.

[63] D.S. Sa, A. Mailis-Gagnon, K. Nicholson, A.E. Lang, Posttraumatic painful torticollis., Mov. Disord. 18 (2003) 1482-91. doi:10.1002/mds.10594.

[64] D.D. Truong, R. Dubinsky, N. Hermanowicz, W.L. Olson, B. Silverman, W.C. Koller, Posttraumatic torticollis., Arch. Neurol. 48 (1991) 221-3.

[65] A.G. Munts, W. Mugge, T.S. Meurs, A.C. Schouten, J. Marinus, G.L. Moseley, F.C. van der Helm, J.J. van Hilten, Fixed Dystonia in Complex Regional Pain Syndrome: a Descriptive and Computational Modeling Approach, BMC Neurol. 11 (2011) 53. doi:10.1186/1471-2377-11-53.

[66] B. Scherokman, F. Husain, A. Cuetter, B. Jabbari, E. Maniglia, Peripheral dystonia., Arch. Neurol. 43 (1986) 830-2. 


\section{Figure captions}

Fig. 1. Pseudodystonic postures of hands in patients with Dupuytren's contracture (A) and scleroderma (B).

Fig. 2. Pseudodystonic posturing of the arms in a patient with Miller-Fisher syndrome.

Fig. 3. Abnormal posture of the left leg in a patient with anti-GAD positive stiff limb syndrome.

Fig. 4. Pseudodystonia of the neck: compensatory neck posture in a patient with left abducens nerve palsy that allows parallel visual axis to avoid diplopia (left). When asked to look to the left, left abducens palsy becomes obvious (right). 


\section{Video legend.}

Video 1. Abnormal movements and postures in a patient with a demyelinating plaque in posterior columns of the cervical spinal cord. Such movements are typically referred to as pseudoathetosis. There is loss of joint position sense and vibration sense in the affected arm. There is worsening of pseudodystonia on eye closure, but no correction on reopening the eyes.

Video 2. Pseudodystonia of both arms in a patient with subacute combined degeneration. Note that worsening of arm posture occurs only after a while when the patient closes his eyes. When walking, the patient looks at his legs to compensate for proprioceptive loss. Romberg's test is positive. Also present when walking are stereotypic money-flipping finger movements of the left hand that may be suggestive of distal sensory loss.

Video 3. Pseudodystonic posturing of the arms in a patient with Miller-Fisher syndrome, with no apparent effect of eye opening/closure. There is also an eye movement disorder, absence of patellar reflexes and ataxia of both arms, more prominent on eye closure.

Video 4. Patient with chronic inflammatory demyelinating polyradiculoneuropathy exhibits pseudodsytonic posturing of the thumbs when counting backwards with eyes closed. Pseudodystonia of both arms on eye closure does not improve when the patient opens his eyes and looks at his arms. Thumb tremor is also present.

Video 5. Abnormal posture of the left leg with superimposed jerks in a patient with anti-GAD positive stiff limb syndrome. 
Video 6. Trousseau sign - hand and arm pseudodystonic posturing and involuntary movements provoked by transient ischaemia in a patient with hypocalcemia, who presented with carpopedal spasms.

Video 7. Patient with typical writer's cramp from age 26, who further developed dystonia with other activities and also at rest and with posture maintenance. Arm dystonia becomes much more apparent after eye closure, even though there are no signs of sensory impairment on detailed clinical examination. MRI of the brain and the cervical spinal cord are normal. 